\title{
Factors Associated with the Efficacy of Radiofrequency Ablation in the Treatment of Benign Thyroid Nodules
}

\author{
Huynh Q Khanh ${ }^{1}$, Nguyen L Vuong ${ }^{2}$, Tran Q Tien ${ }^{3}$
}

\begin{abstract}
Background: Minimally thermal treatments, including radiofrequency ablation (RFA), have been widely used for benign thyroid nodules (BTNs). However, factors related to the efficacy are not consistent among studies. Therefore, this study aimed to investigate factors associated with the efficacy and the risk of having multiple RFA sessions in the treatment of BTNs.

Materials and methods: We performed a retrospective study of 83 patients with 96 BTNs undergoing RFA from 2018 to 2019 . Clinical and ultrasound evaluations were performed before and after RFA 1, 4, 10, and every 6 months afterward. Efficacy outcomes were volume reduction ratio (VRR), symptom and cosmetic scores, and the requirement of multiple RFA sessions.

Results: Female was predominant (86\%) and the median age was 45 years. Most nodules were solid (64\%) with a median volume of $3 \mathrm{~mL}$. Two (2\%) minor complications occurred and median time of follow-up was 17 months. The VRR reached to 56.7, 77, 89.4, and 92.8\% at 4, 10, 16, and 22 months after the RFA, respectively. The symptom and cosmetic scores also significantly reduced. Multiple RFA sessions were required in 14 nodules (15\%). Male had lower VRR of 5.59\% than female, while mixed and cystic nodules had higher VRR of 4.88 and $12.7 \%$ compared with solid nodule. Larger nodule increased the risk of multiple RFA sessions.

Conclusion: Radiofrequency ablation is a safe and effective treatment for BTNs. Gender and solidity associate with VRR, but the large nodule is the risk factor of multiple sessions. More studies are required to reduce the risk of multiple RFA sessions.

Keywords: Goiter, Radiofrequency ablation, Thyroid nodule, Volume reduction ratio.

World Journal of Endocrine Surgery (2020): 10.5005/jp-journals-10002-1309
\end{abstract}

\section{INTRODUCTION}

Thyroid nodules are common all over the world. The prevalence of a palpable thyroid nodule is estimated as 3-7\%, but base on ultrasonography (US), it is estimated that $20-76 \%$ of the general population could be detected as an incidental thyroid nodule. ${ }^{1}$ Although $>90 \%$ of thyroid nodules are benign, ${ }^{2}$ they may pose several issues such as subjective symptoms, jugular oppression, cosmetic problems, and anxiety of malignant transformation, which affect patients' quality of life., ${ }^{1,3}$ Therefore, many benign thyroid nodules (BTNs) require treatment. Previously, there were mostly two choices of treatments for BTNs: surgery and levothyroxine therapy. Currently, levothyroxine is no longer recommended as a single therapy as its modest efficacy but causing iatrogenic hyperthyroidism, ${ }^{4}$ while surgery is limited because of its drawbacks such as a permanent scar, hypothyroidism, and the risk of injury to the recurrent laryngeal nerve. ${ }^{5}$ In recent years, minimally thermal ablation therapies using radiofrequency, laser, and microwave energies have been widely used and have shown good results in the treatment of thyroid nodules. ${ }^{6-11}$

Among several thermal ablation techniques, radiofrequency ablation (RFA) is the most common use for BTNs in many countries. The advantages of RFA are the reduction of complications and hospital stay, preservation of thyroid function, and improvement of the quality of life, in the comparison with surgery. ${ }^{12,13}$ Although the efficacy and safety of RFA have been reported quite consistently in the literature, ${ }^{7-9,11,14}$ one of its disadvantages is the requirement of multiple RFA sessions. Some factors related to the efficacy of RFA in the treatment of thyroid nodules have been reported, including initial volume, US structure, and vascularity of the nodules, ${ }^{15-24}$ but the data are limited and not consistent among studies. Therefore, we performed this study to investigate factors associated with the
${ }^{1}$ Department of Thoracic Surgery, Cho Ray Hospital, Ho Chi Minh City, Vietnam

${ }^{2}$ Department of Cardiovascular and Thoracic Surgery, Faculty of Medicine, University of Medicine and Pharmacy at Ho Chi Minh City, Ho Chi Minh City, Vietnam; Department of Medical Statistics and Informatics, Faculty of Public Health, University of Medicine and Pharmacy at Ho Chi Minh City, Ho Chi Minh City, Vietnam

${ }^{3}$ Department of Thoracic Surgery, Cho Ray Hospital, Ho Chi Minh City, Vietnam; Department of Cardiovascular and Thoracic Surgery, Faculty of Medicine, University of Medicine and Pharmacy at Ho Chi Minh City, Ho Chi Minh City, Vietnam

Corresponding Author: Tran Q Tien, Department of Thoracic Surgery, Cho Ray Hospital, Ho Chi Minh City, Vietnam; Department of Cardiovascular and Thoracic Surgery, Faculty of Medicine, University of Medicine and Pharmacy at Ho Chi Minh City, Ho Chi Minh City, Vietnam, Phone: (+84-028) 3855 4137, e-mail: tranquyettiencr@gmail.com

How to cite this article: Khanh HQ, Vuong NL,TienTQ. Factors Associated with the Efficacy of Radiofrequency Ablation in the Treatment of Benign Thyroid Nodules. World J Endoc Surg 2020;12(3):117-121.

Source of support: Nil

Conflict of interest: None

efficacy of RFA and the requirement of multiple RFA sessions to treat BTNs.

\section{Materials and Methods}

This is a retrospective study of all patients who underwent RFA for BTNs from July 2018 to August 2019 at the Thoracic Surgery Department of a tertiary medical center in Ho Chi Minh City, Vietnam. 
The study was approved by the institutional ethics committee. The inclusion criteria were (1) evidence of BTN(s) by cytology using two separate US-guided fine-needle aspiration (US-FNA) biopsies and (2) undergoing RFA therapy to treat the nodule(s). We excluded all patients with (1) nodule that was suspicious of malignancy on US findings such as noticeable microcalcifications, marked hypoechoic, or ill-defined margins, and (2) previously undergoing other ablation therapy for thyroid nodule (e.g., microwave ablation).

\section{Radiofrequency Ablation Procedure}

All patients underwent the RFA procedure in a supine position with mild extended neck in aseptic conditions, under US guidance. First, we performed local anesthesia at the puncture site and then dissected the thyroid gland and subcutaneous tissues using $10 \mathrm{~mL}$ of $1 \%$ lidocaine. Then, we inserted an 18-gauge internally cooled monopolar RFA electrode with 7-mm active tip into the thyroid nodule using the "trans-isthmic" approach to minimize the risk of injury to the recurrent laryngeal nerve. ${ }^{25,26}$ The electrode was connected to a radiofrequency generator (Mygen V-1000, RF Medical Inc.) which could deliver up to 140 -Watt power. In case of mixed or cystic nodule, we aspirated the whole internal fluid before ablation. We then performed the ablation using the "moving-shot" technique ${ }^{25,26}$ with a $30-50$-Watt power until the whole nodule became a transient hyperechoic zone. During the ablation, we paid attention to preventing the injury to the surrounding important structures, such as the laryngeal nerve, carotid vessels, esophagus, and trachea. After the procedure, patients were under observation for 1-2 hours and were discharged if having no severe pain or complications. Oral analgesic with acetaminophen was prescribed for three days.

\section{Assessment of Patients and Definition of Outcomes}

Clinical and US evaluations were performed before and after the RFA 1, 4, 10, and every 6 months afterward. Clinical examinations included the evaluation of the symptom and cosmetic scores. ${ }^{26}$ The symptom score was obtained from patients using a visual analog scale of 0 to 10 by asking the question "To what degree did the thyroid nodule(s) affect you in terms of cosmetic, functional, or psychological problem?". Higher scores represent greater distress of patients by symptoms. The cosmetic score was evaluated by the doctor and had four grades: grade 0 was no palpable mass, grade I was a palpable but invisible mass, grade II was a visible mass when extending the neck or swallowing, and grade III was an easily visible mass. In the US examination, we evaluated the diameters, volume, echogenicity, solidity, number, location, and the signs of suspicious malignancy of the thyroid nodule(s). The volume of a nodule was calculated from the three orthogonal diameters using the equation $V=\pi a b c / 6(V$ is the volume in $\mathrm{mL} ; a, b$, and $c$ are the three diameters in $\mathrm{cm}$; and $\pi$ is 3.14). The solidity of the nodules was classified into three categories: solid when the solid component was $>75 \%$; cystic when the solid component was $<25 \%$; and mixed was in-between.

Complications and side effects were assessed during and after the procedure and classified into minor and major complications as recommended by the international working group on imageguided tumor ablation. ${ }^{27}$ A complication was classified as major if it led to increasing the level of care, caused substantial disability, or was fatal. Other complications were minor.

The efficacy of the RFA was mainly evaluated using the volume reduction ratio (VRR) of the treated thyroid nodule, which is the percentage of volume reduction during the follow-up period compared to the initial volume before ablation:
$\operatorname{VRR}(\%)=100 \% \times\left(V_{0}-V_{x}\right) / V_{0}\left(V R R: V R R\right.$ in $\% ; V_{0}$ : initial volume before ablation in $\mathrm{mL} ; V_{x}$ : volume at follow-up in $\mathrm{mL}$ ). Other parameters to evaluate the efficacy were the symptom and cosmetic scores, and the requirement of multiple RFA sessions. The indication of an additional RFA after the first ablation was a low VRR during the follow-up period (VRR was $<25 \%$ at 1 month, or $<50 \%$ at 6 months, or $<75 \%$ at 12 months) in combination with an evidence of a viable portion inside the nodule on the US examination (same echogenicity with that nodule before any ablation, or the presence of vascularity inside the nodule).

\section{Statistical Analysis}

Characteristics of the patients and all treated thyroid nodules were summarized by count and percentage for categorical variables, and median and interquartile range (IQR) for continuous variables. The efficacy outcomes (VRR and symptom and cosmetic scores) were reported before the RFA and at each time-point of follow-up using median and IQR. To find factors associated with the VRR, uni- and multivariable linear mixed-effects models were used to account for the correlated data from multiple nodules of a patient and repeated measurements of VRR for a nodule. We performed the univariable model for each factor of gender, age, time from diagnosis to the first RFA, comorbidities, initial volume and solidity of the nodule, and the requirement of multiple RFA sessions. A multivariable model including all of these co-variables was then performed. Results of the models were expressed by the mean difference (MD) of the VRR, 95\% confidence interval $(\mathrm{Cl})$, and $p$ value. In addition, we compared the characteristics of patients and nodules before ablation of the two groups (group required only one RFA session and group required multiple RFA sessions) using Fisher's exact test for categorical variables and Mann-Whitney $U$ test for continuous variables. Finally, a multivariable logistic regression model was used to find factors associated with the requirement of multiple RFA sessions. All $p$ values were two-sided and $p$ values of $<0.05$ defined statistical significance. All analyses were performed using the statistical software $R$ version 3.6.3.

\section{Results}

\section{Patient's Characteristics}

A total of 83 patients with 96 BTNs were included in the study. Female was predominant (86\%) and the median age was 45 years. The median time from thyroid nodule diagnosis to the first RFA was eight months. Seventeen patients (20\%) had comorbidities with most were hypertension. Patients had high distress with a median symptom score of seven (IQR: 5-8). Regarding the cosmetic score, $59 \%$ of all patients had a visible mass: 21 cases (25\%) with grade II and 28 cases (34\%) with grade III. There were 13 patients (16\%) with two treated nodules. All patients underwent a safe RFA procedure with only two (2\%) minor complications occurred: one with small hematoma at the puncture site and the other with temporary voice change. Both recovered in 1 week after the procedure. The median time of follow-up was 17 months (Table 1).

\section{Nodule's Characteristics}

The median largest diameter and median volume of all treated thyroid nodules were $26 \mathrm{~mm}$ and $3 \mathrm{~mL}$, respectively. The majority were isoechoic $(61 \%)$ or spongiform (25\%). There were 61 nodules (64\%) classified as solid, 26 nodules (27\%) as mixed, and 9 nodules (9\%) as cystic. Most of the nodules required one RFA session, but 14 nodules (15\%) required multiple sessions (Table 2 ). 
Table 1: Clinical features of patients

\begin{tabular}{lc}
\hline & Summary statistic $(\mathrm{N}=83)$ \\
\hline Gender female & $71(86)$ \\
Age (years) & $45(36-54)$ \\
Time from diagnosis to first RFA (months) & $8(6-10)$ \\
Comorbidities & $17(20)$ \\
- Hypertension & $16(19)$ \\
- Diabetes & $3(4)$ \\
Symptom score & $7(5-8)$ \\
Cosmetic score & \\
- Grade 0 & $2(2)$ \\
- Grade I & $32(39)$ \\
- Grade II & $21(25)$ \\
- Grade III & $28(34)$ \\
Number of treated nodules & \\
- 1 Nodule & $70(84)$ \\
- 2 Nodules & $13(16)$ \\
Complication & \\
- Major complications & $0(0)$ \\
- Minor complications & $2(2)$ \\
Follow-up period (months) & $17(15-22)$ \\
\hline
\end{tabular}

Summary statistic is the number of patients and percentage for categorical variables and median (interquartile range) for continuous variables RFA, radiofrequency ablation

Table 2: Characteristics of the treated thyroid nodules

\begin{tabular}{ll}
\hline & Summary statistic $(N=96)$ \\
\hline $\begin{array}{l}\text { Largest diameter }(\mathrm{mm}) \\
\text { Echogenicity }\end{array}$ & $3(18-10)$ \\
- Isoechoic & $59(61)$ \\
- Spongiform or partially cystic & $24(25)$ \\
- Hyperechoic & $13(14)$ \\
Solidity & \\
- Solid & $61(64)$ \\
- Mixed & $26(27)$ \\
- Cystic & $9(9)$ \\
Location & \\
- Right lobe & $51(53)$ \\
- Left lobe & $45(47)$ \\
Number of RFA sessions & \\
- 1 Session & $82(85)$ \\
- 2 Sessions & $12(13)$ \\
- 3 Sessions & $2(2)$
\end{tabular}

Summary statistic is a number of patients and percentage for categorical variables and median (interquartile range) for continuous variables RFA, radiofrequency ablation

\section{Factors Related to the Efficacy Outcomes}

Table 3 shows the efficacy of RFA in our study. The VRR increased during the follow-up period, reached to $37.1 \%$ (IQR: $31.0-43.5 \%$ ) at 1 month, 56.7\% (IQR: 50.1-63.4\%) at 4 months, 77\% (IQR: 74.9-78.9\%) at 10 months, and approximately $90 \%$ at 16 and 22 months after the ablation. The symptom and cosmetic scores also significantly reduced and were nearly zero after 1 year.

In the univariable analyses, significant factors associated with the VRR were gender, comorbidities, initial volume and solidity of the nodules, and multiple RFA sessions. However, in the multivariable analysis, only gender and solidity of the nodules were associated with the VRR: male had lower VRR of 5.59\% (95\% Cl:0.64-10.54\%) compared to female, and mixed and cystic nodules had higher VRR than solid nodule of 4.88 (1.02-8.75) and 12.7 (6.66-18.75) \%, respectively (Table 4).

Compared to the group with one RFA session, the multiple RFA sessions group had a significantly longer time from diagnosis to the first RFA (median: 12 vs 8 months), higher symptom and cosmetic scores, significantly larger thyroid nodules with higher diameter (median: 48 vs $20 \mathrm{~mm}$ ) and volume (median: 28 vs $2 \mathrm{~mL}$ ) (Table 5). In the multivariable model, after correcting for time of diagnosis and solidity, initial volume of the treated nodules significantly increased the risk of requiring multiple RFA sessions [OR $(95 \% \mathrm{Cl})$ : $1.23(1.12-1.42)]$ (Table 6).

\section{Discussion}

This study shows the safety and efficacy of RFA in the treatment of BTNs. Only two minor complications occurred and no major complication was found. The volume of the nodules reduced by $37.1,56.7,77$, and around $90 \%$ at $1,4,10$, and $\geq 16$ months after the ablation. We also found that gender male had lower VRR than female and more cystic of the nodules associated with the higher VRR during following up. There were $15 \%$ of cases requiring multiple RFA sessions and the initial volume of the nodules was the significantly associated factor: larger nodule leads to a higher risk of the requirement of multiple RFA sessions.

Radiofrequency ablation has been applied in the treatment of BTNs in many countries. Several studies have evaluated its efficacy and have shown that the volume reduction of treated nodules reached approximately $>60 \%$ at 6 months, $>70 \%$ at 12 months, and $>80 \%$ at 24 months after the ablation. $8,9,11,14$ The variation of the results between different studies might be due to different types and sizes of the nodules as well as a variety of instruments and techniques used. Our study showed similar results to others, of which the volume reduction increased rapidly within the first 4 months after the procedure and then steadily rose thereafter. The significant reduction of the nodule's volume within the first 4 months helps to solve the clinical symptoms and cosmetic concerns of patients caused by the nodule(s), which is demonstrated in our study that the symptom and cosmetic scores significantly decreased after the ablation. In addition, RFA is safe as having a very low rate of complications, particularly while comparing with surgery. ${ }^{13,14}$

Table 3: Efficacy outcomes during the follow-up period

\begin{tabular}{lllllll}
\hline Outcome & Before RFA & 1 Month & 4 Months & 10 Months & 16 Months & 22 Months \\
\hline VRR $(\%)$ & - & $37.1(31.0 ; 43.5)$ & $56.7(50.1 ; 63.4)$ & $77.0(74.9 ; 78.9)$ & $89.4(84.2 ; 93.9)$ & $92.8(86.5 ; 96.7)$ \\
Symptom score & $7(5 ; 8)$ & $4(4 ; 5)$ & $3(2 ; 3)$ & $2(0 ; 2)$ & $0(0 ; 2)$ & $0(0 ; 0)$ \\
Cosmetic score & $2(1 ; 3)$ & $1(1 ; 2)$ & $1(1 ; 2)$ & $1(0 ; 1)$ & $0(0 ; 1)$ & $0(0 ; 0)$ \\
\hline
\end{tabular}

Summary statistic is median (interquartile range)

RFA, radiofrequency ablation; VRR, volume reduction ratio 
Table 4: Factors associated with volume reduction ratio

\begin{tabular}{|c|c|c|c|c|c|c|}
\hline \multirow[b]{2}{*}{ Factor } & \multicolumn{3}{|c|}{ Univariable analysis } & \multicolumn{3}{|c|}{ Multivariable analysis } \\
\hline & $M D$ & $95 \% \mathrm{Cl}$ & $p$ value & $M D$ & $95 \% \mathrm{Cl}$ & $p$ value \\
\hline Gender male & -5.78 & $-10.92 ;-0.65$ & 0.029 & -5.59 & $-10.54 ;-0.64$ & 0.027 \\
\hline Age (per 1-year increase) & 0.12 & $-0.02 ; 0.26$ & 0.090 & 0.04 & $-0.15 ; 0.22$ & 0.696 \\
\hline Time from diagnosis to first RFA (per 1-month increase) & -0.26 & $-0.70 ; 0.17$ & 0.235 & -0.09 & $-0.62 ; 0.43$ & 0.724 \\
\hline Comorbidities (yes) & 5.30 & $1.15 ; 9.44$ & 0.014 & 4.53 & $-0.74 ; 9.80$ & 0.093 \\
\hline Initial volume (per 1-mL increase) & -0.14 & $-0.26 ;-0.03$ & 0.017 & -0.10 & $-0.27 ; 0.07$ & 0.250 \\
\hline \multicolumn{7}{|l|}{ Solidity } \\
\hline - Solid & Ref & Ref & Ref & Ref & Ref & Ref \\
\hline - Mixed & 3.98 & $0.15 ; 7.81$ & 0.044 & 4.88 & $1.02 ; 8.75$ & 0.014 \\
\hline - Cystic & 12.56 & $6.77 ; 18.36$ & $<0.001$ & 12.70 & $6.66 ; 18.75$ & $<0.001$ \\
\hline Multiple RFA sessions & -7.61 & $-12.00 ;-3.22$ & $<0.001$ & -4.05 & $-10.41 ; 2.30$ & 0.212 \\
\hline
\end{tabular}

$\mathrm{Cl}$, confidence interval; $\mathrm{MD}$, mean difference; Ref, reference

Table 5: Characteristics across groups regarding the number of microwave ablation compared sessions

\begin{tabular}{|c|c|c|c|}
\hline Characteristic & $\begin{array}{l}1 \text { RFA session } \\
(N=82)\end{array}$ & $\begin{array}{l}>1 \text { RFA sessions } \\
(N=14)\end{array}$ & $p$ value \\
\hline Gender female & $72(88)$ & $12(86)$ & 0.686 \\
\hline Age (years) & $46(36-56)$ & $43(40-52)$ & 0.506 \\
\hline $\begin{array}{l}\text { Time from diagnosis to } \\
\text { first RFA (months) }\end{array}$ & $8(6-10)$ & $12(7-12)$ & 0.014 \\
\hline Comorbidities & $19(23)$ & $2(14)$ & 0.728 \\
\hline Symptom score & $6(5-7)$ & $10(9-10)$ & $<0.001$ \\
\hline Cosmetic score & & & $<0.001$ \\
\hline - Grade 0 & $2(2)$ & $0(0)$ & \\
\hline - Grade I & $34(41)$ & $0(0)$ & \\
\hline - Grade II & $26(32)$ & $1(7)$ & \\
\hline - Grade III & $20(24)$ & $13(93)$ & \\
\hline Complication (yes) & $2(2)$ & $0(0)$ & 1.000 \\
\hline Largest diameter (mm) & $20(18-30)^{\prime}$ & $48(40-54)$ & $<0.001$ \\
\hline Volume $(\mathrm{mL})$ & $2(1-5)$ & $28(12-50)$ & $<0.001$ \\
\hline Echogenicity & & & 0.537 \\
\hline - Isoechoic & $52(63)$ & $7(50)$ & \\
\hline $\begin{array}{l}\text { - Spongiform or } \\
\text { partially cystic }\end{array}$ & $19(23)$ & $5(36)$ & \\
\hline - Hyperechoic & $11(13)$ & $2(14)$ & \\
\hline Solidity & & & 0.814 \\
\hline - Solid & $53(65)$ & $8(57)$ & \\
\hline - Mixed & $21(26)$ & $5(36)$ & \\
\hline - Cystic & $8(10)$ & $1(7)$ & \\
\hline
\end{tabular}

Summary statistic is a number of nodules and percentage for categorical variables and median (interquartile range) for continuous variables. $p$ values were calculated based on Fisher's exact test (categorical data) and Mann-Whitney $U$ test (continuous data). RFA, radiofrequency ablation

Several potential complications of RFA are bleeding due to nodule rupture while performing the procedure, injury to the recurrent laryngeal nerve, or other important structures (carotid vessels, trachea, and esophagus), skin burn, and infection. Currently, with the support of US guidance and internally cooled technology, RFA can be performed safely. Major complications are infrequent and
Table 6: Factors associated with multiple RFA sessions

\begin{tabular}{|c|c|c|c|}
\hline Factor & OR & $95 \% \mathrm{Cl}$ & $p$ value \\
\hline $\begin{array}{l}\text { Time from diagnosis to first RFA } \\
\text { (months) }\end{array}$ & 1.13 & $0.93 ; 1.37$ & 0.185 \\
\hline Initial volume (per 1-mL increase) & 1.23 & $1.12 ; 1.42$ & $<0.001$ \\
\hline \multicolumn{4}{|l|}{ Solidity } \\
\hline - Solid & Ref & Ref & Ref \\
\hline - Mixed & 0.37 & $0.03 ; 2.69$ & 0.371 \\
\hline - Cystic & 0.10 & $0.01 ; 6.15$ & 0.111 \\
\hline
\end{tabular}

$\mathrm{Cl}$, confidence interval; Ref, reference

the rate of minor complications is almost $<10 \%$. ${ }^{8,9,11,14}$ Currently, RFA and other minimally thermal ablations are applied for the treatment of papillary thyroid carcinoma as well. ${ }^{7}$

A problem of RFA is the requirement of multiple ablation sessions. The reason is that the volume is slowly reduced or even remained or increased during the follow-up period. This might be due to the incomplete ablation or different effects of RFA in different types of thyroid nodules. In our study, we found that gender and the initial solidity of nodules are significantly associated with the VRR. We could not explain why males had lower VRR than females, this may be because of the relatively small sample size that limits the generalization of this result, thus a larger study is needed to confirm this. However, the association between the solidity of thyroid nodules and VRR has been demonstrated in other studies. ${ }^{15-18,20-24}$ The more cystic of the nodules leads to higher VRR, which is explained by the aspiration of cystic fluid inside the nodules during the RFA procedure. Although gender and solidity of the nodules were significantly associated with VRR, they showed no association with the risk of having multiple RFA sessions in our study. We found only one risk factor of having multiple RFA sessions which is the initial size of the nodules represented by the initial volume: larger nodule has a higher risk of having multiple RFA sessions. Some other studies showed similar results and the reason may be the incomplete ablation in the case of a large nodule. ${ }^{23,24}$ This result would help clinicians to forecast the risk of multiple sessions before performing RFA to treat BTNs.

Limitations of the study are the retrospective single-center design with relatively small sample size and the absence of histological confirmation of BTNs which is similar to other 
studies without surgery. However, we enrolled patients with the confirmation of benign nodules based on two separate US-FNA cytology results and no suspicion of malignancy on US findings. We also followed up with patients regularly and found no malignant nodules later on.

\section{Conclusion}

Radiofrequency ablation is a safe and effective treatment for BTNs. The median VRR of the treated nodules reached 56.7, 77, 89.4 , and $92.8 \%$ at $4,10,16$, and 22 months after the procedure. Both symptom and cosmetic concerns significantly improved accordingly, while no major complications occurred. We found that gender and solidity of the nodules were associated with VRR, but a large nodule increased the risk of having multiple RFA sessions. Future studies are required to better determine associated factors of the efficacy of RFA and reduce the risk of multiple RFA sessions.

\section{References}

1. Gharib H, Papini E, Paschke R, et al. American Association of Clinical Endocrinologists, Associazione Medici Endocrinologi, and European Thyroid Association Medical Guidelines for Clinical Practice for the diagnosis and management of thyroid nodules. J Endocrinol Invest 2010;33(5 Suppl):1-50. DOI: 10.1007/BF03346587.

2. Hegedus L. Clinical practice. The thyroid nodule. N Engl J Med 2004;351(17):1764-1771. DOI: 10.1056/NEJMcp031436.

3. Arora N, Scognamiglio T, Zhu B, et al. Do benign thyroid nodules have malignant potential? An evidence-based review. World J Surg 2008;32(7):1237-1246. DOI: 10.1007/s00268-008-9484-1.

4. Haugen BR, Alexander EK, Bible KC, et al. 2015 American Thyroid Association Management Guidelines for adult patients with thyroid nodules and differentiated thyroid cancer: the American thyroid association guidelines task force on thyroid nodules and differentiated thyroid cancer. Thyroid 2016;26(1):1-133. DOI: 10.1089/ thy.2015.0020.

5. Bandeira-Echtler E, Bergerhoff K, Richter B. Levothyroxine or minimally invasive therapies for benign thyroid nodules. Cochrane Database Syst Rev 2014(6):CD004098. DOI: 10.1002/14651858. CD004098.pub2.

6. Mainini AP, Monaco C, Pescatori LC, et al. Image-guided thermal ablation of benign thyroid nodules. J Ultrasound 2017;20(1):11-22. DOI: 10.1007/s40477-016-0221-6.

7. Tong M, LiS, LiY, et al. Efficacy and safety of radiofrequency, microwave and laser ablation for treating papillary thyroid microcarcinoma: a systematic review and meta-analysis. Int J Hyperthermia 2019;36(1):1278-1286. DOI: 10.1080/02656736.2019.1700559.

8. Cho SJ, Baek JH, Chung SR, et al. Long-term results of thermal ablation of benign thyroid nodules: a systematic review and metaanalysis. Endocrinol Metab (Seoul) 2020;35(2):339-350. DOI: 10.3803/ EnM.2020.35.2.339.

9. Kim HJ, Cho SJ, Baek JH, et al. Efficacy and safety of thermal ablation for autonomously functioning thyroid nodules: a systematic review and meta-analysis. Eur Radiol 2020;31(2):605-615. DOI: 10.1007/ s00330-020-07166-0.

10. Papini E, Monpeyssen H, Frasoldati A, et al. 2020 European Thyroid Association Clinical Practice Guideline for the use of image-guided ablation in benign thyroid nodules. Eur Thyroid J 2020;9(4):172-185. DOI: 10.1159/000508484.
11. Trimboli P, Castellana M, Sconfienza LM, et al. Efficacy of thermal ablation in benign non-functioning solid thyroid nodule: a systematic review and meta-analysis. Endocrine 2020;67(1):35-43. DOI: 10.1007/ s12020-019-02019-3.

12. Che $Y$, Jin $S$, Shi $C$, et al. Treatment of benign thyroid nodules: comparison of surgery with radiofrequency ablation. AJNR Am J Neuroradiol 2015;36(7):1321-1325. DOI: 10.3174/ajnr.A4276.

13. Yue WW, Wang SR, Lu F, et al. Quality of life and cost-effectiveness of radiofrequency ablation versus open surgery for benign thyroid nodules: a retrospective cohort study. Sci Rep 2016;6(1):37838. DOI: 10.1038/srep37838.

14. Chung SR, Suh $\mathrm{CH}$, Baek JH, et al. Safety of radiofrequency ablation of benign thyroid nodules and recurrent thyroid cancers: a systematic review and meta-analysis. Int J Hyperthermia 2017;33(8):920-930. DOI: 10.1080/02656736.2017.1337936.

15. Cesareo R, Naciu AM, lozzino M, et al. Nodule size as predictive factor of efficacy of radiofrequency ablation in treating autonomously functioning thyroid nodules. Int J Hyperthermia 2018;34(5):617-623. DOI: 10.1080/02656736.2018.1430868.

16. Deandrea M, Garino F, Alberto $M$, et al. Radiofrequency ablation for benign thyroid nodules according to different US features: an Italian multicentre prospective study. Eur J Endocrinol 2019;180(1):79-87. DOI: 10.1530/EJE-18-0685.

17. Dobnig $\mathrm{H}$, Amrein $\mathrm{K}$. Monopolar radiofrequency ablation of thyroid nodules: a prospective Austrian single-center study. Thyroid 2018;28(4):472-480. DOI: 10.1089/thy.2017.0547.

18. Jung SL, Baek JH, Lee JH, et al. Efficacy and safety of radiofrequency ablation for benign thyroid nodules: a prospective multicenter study. Korean J Radiol 2018;19(1):167-174. DOI: 10.3348/kjr.2018.19.1.167.

19. Lee GM, You JY, Kim HY, et al. Successful radiofrequency ablation strategies for benign thyroid nodules. Endocrine 2019;64(2):316-321. DOI: 10.1007/s12020-018-1829-4.

20. Sim JS, Baek JH, Cho W. Initial ablation ratio: quantitative value predicting the therapeutic success of thyroid radiofrequency ablation. Thyroid 2018;28(11):1443-1449. DOI: 10.1089/thy.2018.0180.

21. Vuong NL, Dinh LQ, Bang HT, et al. Radiofrequency ablation for benign thyroid nodules: 1-year follow-up in 184 patients. World J Surg 2019;43(10):2447-2453. DOI: 10.1007/s00268-019-05044-5.

22. Bernardi S, Giudici F, Cesareo R, et al. Five-year results of radiofrequency and laser ablation of benign thyroid nodules: a multicenter study from the Italian minimally invasive treatments of the thyroid group. Thyroid 2020;30(12):1759-1770. DOI: 10.1089/ thy.2020.0202.

23. Feroci F, Guagni T, Coppola A, et al. Radiofrequency thermal ablation of benign thyroid nodules: the correlation between ultrasound nodule characteristics and results. Surg Innov 2020;27(4):342-351. DOI: $10.1177 / 1553350620913134$.

24. Yan L, Luo Y, Xie F, et al. Residual vital ratio: predicting regrowth after radiofrequency ablation for benign thyroid nodules. Int J Hyperthermia 2020;37(1):1139-1148. DOI: 10.1080/02656736.2020.1825835.

25. Baek JH, Moon WJ, Kim YS, et al. Radiofrequency ablation for the treatment of autonomously functioning thyroid nodules. World J Surg 2009;33(9):1971-1977. DOI: 10.1007/s00268-009-0130-3.

26. Baek JH, Kim YS, Lee D, et al. Benign predominantly solid thyroid nodules: prospective study of efficacy of sonographically guided radiofrequency ablation versus control condition. AJR Am J Roentgenol 2010;194(4):1137-1142. DOI: 10.2214/AJR.09.3372.

27. Ahmed M, Solbiati L, Brace CL, et al. Image-guided tumor ablation: standardization of terminology and reporting criteria--a 10-year update. Radiology 2014;273(1):241-260. DOI: 10.1148/radiol.14132958. 